\title{
ORDER OF GROWTH OF SOLUTIONS TO ALGEBRAIC DIFFERENTIAL EQUATIONS IN THE UNIT DISK
}

\author{
D. BENBOURENANE and L. R. SONS
}

Received 3 February 2004

\begin{abstract}
S. B. Bank has shown that there is no uniform growth estimate for meromorphic solutions of algebraic differential equations with meromorphic coefficients in the unit disk. We give conditions under which such solutions must have a finite order of growth.
\end{abstract}

2000 Mathematics Subject Classification: 30D35.

1. Introduction. Consider the algebraic differential equation

$$
\sum_{\bar{\alpha} \in I} a_{\bar{\alpha}}(z) y^{\alpha_{0}}\left(y^{\prime}\right)^{\alpha_{1}} \cdots\left(y^{(k)}\right)^{\alpha_{k}}=0,
$$

where $I$ is a finite set of distinct tuples $\left(\alpha_{0}, \alpha_{1}, \ldots, \alpha_{k}\right)$ for which each $\alpha_{i}$ is a nonnegative integer, and the $a_{\bar{\alpha}}$ are meromorphic functions in $D=\{z|| z \mid<1\}$. For some index sets $I$, we determine conditions on $a_{\bar{\alpha}}$, whereby a meromorphic solution $f$ of (1.1) in $D$ will have finite order of growth as measured by the Ahlfors-Shimizu characteristic function.

In [1], Bank investigated (1.1) where $I$ consists of 2-tuples and the $a_{\bar{\alpha}}$ are arbitrary analytic functions of finite order in the unit disk. He observed that such equations could possess analytic solutions of infinite order in the unit disk, but obtained a uniform growth estimate for all such solutions. He further noted that for arbitrary meromorphic solutions in the disk, no such uniform growth estimate is possible.

Recently, Heittokangas [3] showed for certain sets $I$ that each meromorphic solution of (1.1) has finite order when the $a_{\bar{\alpha}}$ are polynomial functions. Further, he and Wulan [5] studied the equation

$$
\left(y^{\prime}\right)^{n}=\sum_{\bar{\alpha} \in I} b_{\bar{\alpha}}(z) y^{\alpha_{0}}\left(y^{\prime}\right)^{\alpha_{1}} \cdots\left(y^{(k)}\right)^{\alpha_{k}},
$$

where each $b_{\bar{\alpha}}$ is analytic in $D$ and satisfies $\sup _{z \in D}\left(1-|z|^{2}\right)^{q}\left|b_{\bar{\alpha}}(z)\right|<\infty$ for some $q \geq 0$, and showed that if $n$ is large enough relative to the size of the number $q$, then each meromorphic solution of (1.2) has finite order. Our first theorem is similar in character to the result of Wulan and Heittokangas, while our other theorems take into account the nature of the zeros or poles of the $a_{\bar{\alpha}}$ coefficient functions.

\section{Statement of results}

THEOREM 2.1. Let $f$ be a meromorphic function in the unit disk $D$ which satisfies a differential equation of the form (1.1), where the sum is taken over some finite index set I 
of distinct $m$-tuples $\bar{\alpha}=\left(\alpha_{0}, \alpha_{1}, \ldots, \alpha_{m}\right)$ for which each $\alpha_{i}$ is a nonnegative integer and the $a_{\bar{\alpha}}$ are meromorphic functions in D. Suppose that

(i) there exists a $\bar{\beta} \in I$, where $\beta_{1} \geq 1$ such that $a_{\bar{\beta}}$ is not identically zero,

(ii) for all $\bar{\alpha} \in I \backslash\{\bar{\beta}\}, q=\left(\beta_{1}-\alpha_{1}\right)+2\left(\beta_{2}-\alpha_{2}\right)+\cdots+m\left(\beta_{m}-\alpha_{m}\right)$ is a positive integer,

(iii) for all $\bar{\alpha} \in I \backslash\{\bar{\beta}\}$ and for all $z \in D$,

$$
\left|\frac{a_{\bar{\alpha}}(z)}{a_{\bar{\beta}}(z)}\right|=O\left(\frac{1}{(1-|z|)^{q}}\right) .
$$

Then

$$
\limsup _{r \rightarrow 1} \frac{\log T_{0}(r, f)}{-\log (1-r)}<\infty
$$

where $T_{0}$ denotes the Ahlfors-Shimizu characteristic function.

Our second result concerns the situation where a restriction is placed on the number of poles each coefficient function can have. We use the usual little $n$ counting function of Nevanlinna theory and state the following theorem.

THEOREM 2.2. Let $f$ be a meromorphic function in the unit disk $D$ which satisfies a differential equation of the form (1.1), where the sum is taken over some finite index set I of distinct $m$-tuples $\bar{\alpha}=\left(\alpha_{0}, \alpha_{1}, \ldots, \alpha_{m}\right)$ for which each $\alpha_{i}$ is a nonnegative integer and the $a_{\bar{\alpha}}$ are meromorphic functions in D. Suppose that

(i) for each $\bar{\alpha} \in I, \int_{0}^{1}(1-r) n\left(r, a_{\bar{\alpha}}\right) d r<\infty$,

(ii) there exists a $\bar{\beta} \in I$, where $\beta_{1} \geq 1$ such that $a_{\bar{\beta}}$ is not identically zero and $\int_{0}^{1}(1-$ $r) n\left(r, 1 / a_{\bar{\beta}}\right) d r<\infty$,

(iii) for all $\bar{\alpha} \in I \backslash\{\bar{\beta}\}, q=\left(\beta_{1}-\alpha_{1}\right)+2\left(\beta_{2}-\alpha_{2}\right)+\cdots+m\left(\beta_{m}-\alpha_{m}\right)$ is positive,

(iv) for all $\bar{\alpha} \in I \backslash\{\bar{\beta}\}$ and for all $z \in D$,

$$
\left|\frac{h_{\bar{\alpha}}(z)}{h_{\bar{\beta}}(z)}\right|=O\left(\frac{1}{(1-|z|)^{q}}\right),
$$

where $a_{\bar{\alpha}}(z)=h_{\bar{\alpha}}(z) / z^{l(\bar{\alpha})} P_{\bar{\beta}}(z)$ with $P_{\bar{\alpha}}(z)$ the Blaschke product for the poles of $a_{\bar{\alpha}}$ and $a_{\bar{\beta}}(z)=z^{l(\bar{\beta})} P_{\bar{\beta}}(z) h_{\bar{\beta}}(z)$ with $P_{\bar{\beta}}(z)$ the Blaschke product for the zeros of $a_{\bar{\beta}}$.

Then

$$
\limsup _{r \rightarrow 1} \frac{\log T_{0}(r, f)}{-\log (1-r)}<\infty
$$

Our third result allows the coefficient functions $a_{\bar{\alpha}}$ of (1.1) to have more poles than Theorem 2.2 does. To state it easily, we need to recall some facts and terms concerning the canonical products in the unit disk introduced by Tsuji [4]. 
If $f$ is a meromorphic function of finite order $\sigma$ in the unit disk with $\left\{a_{n}\right\}$ its zero points for which $a_{n} \neq 0$, then $\sum_{k}\left(1-\left|a_{n}(a)\right|\right)^{\sigma+1+\varepsilon}<\infty$ for each $\varepsilon>0$.

The convergence exponent $\mu \geq 0$ of $\left\{\left|a_{n}\right|\right\}$ is defined to be zero if $\sum_{n}\left(1-\left|a_{n}\right|\right)<\infty$, whereas otherwise it is that number for which

$$
\sum_{k}\left(1-\left|a_{n}(a)\right|\right)^{\mu+1-\varepsilon}=\infty, \quad \sum_{k}\left(1-\left|a_{n}(a)\right|\right)^{\mu+1+\varepsilon}<\infty
$$

for any $\varepsilon>0$. It follows that $0 \leq \mu \leq \sigma$.

The Tsuji canonical product $P$ formed with $\left\{a_{n}\right\}$ is defined by

$$
P(z)=\prod_{n=1}^{\infty}\left(1-\frac{1-\left|a_{n}\right|^{2}}{1-\overline{a_{n}} z}\right)
$$

when $\sum_{n}\left(1-\left|a_{n}\right|\right)<\infty$, while

$$
P(z)=\prod_{n=1}^{\infty}\left(1-\frac{1-\left|a_{n}\right|^{2}}{1-\overline{a_{n}} z}\right) \exp \left\{\frac{1-\left|a_{n}\right|^{2}}{1-\overline{a_{n}} z}+\frac{1}{2}\left(\frac{1-\left|a_{n}\right|^{2}}{1-\overline{a_{n}} z}\right)^{2}+\cdots+\frac{1}{p}\left(\frac{1-\left|a_{n}\right|^{2}}{1-\overline{a_{n}} z}\right)^{p}\right\}
$$

when $\sum_{n}\left(1-\left|a_{n}\right|\right)=\infty$ with $p$ a positive integer satisfying $\sum_{n}\left(1-\left|a_{n}\right|\right)^{p}=\infty$ and $\sum_{n}\left(1-\left|a_{n}\right|\right)^{p+1}<\infty$.

It follows that $p-1 \leq \mu$. Further, Tsuji [4, page 227] has shown that when $\sum_{n}(1-$ $\left.\left|a_{n}\right|\right)=\infty$, the order of $P$ is equal to $\mu$.

THEOREM 2.3. Let $f$ be a meromorphic function in the unit disk $D$ which satisfies a differential equation of the form (1.1), where the sum is taken over some finite index set I of distinct $m$-tuples $\bar{\alpha}=\left(\alpha_{0}, \alpha_{1}, \ldots, \alpha_{m}\right)$ for which each $\alpha_{i}$ is a nonnegative integer and the $a_{\bar{\alpha}}$ are meromorphic functions in $D$. Assume that

(i) there exists a $\bar{\beta} \in I$, where $\beta_{1} \geq 1$ and $a_{\bar{\beta}}$ is not identically zero,

(ii) for all $\bar{\alpha} \in I \backslash\{\bar{\beta}\}, q=\left(\beta_{1}-\alpha_{1}\right)+2\left(\beta_{2}-\alpha_{2}\right)+\cdots+m\left(\beta_{m}-\alpha_{m}\right)$ is a positive integer with $q>K\left(\hat{\mu}+v_{\beta}+6\right)$, where $v_{\beta}$ is the convergence exponent for the zeros of the zeros of $1 / a \beta, \hat{\mu}=\max _{\bar{\alpha} \in I \backslash\{\beta\}} \mu_{\bar{\alpha}}$ with $\mu_{\bar{\alpha}}$ the convergence exponent for the zeros of $a_{\bar{\alpha}}$, and

$K=\max \left(8^{p_{\bar{\beta}}+1} \sum_{i=1}^{\infty}\left(1-\left|c_{i, \bar{\beta}}\right|\right)^{p_{\bar{\beta}}+1}, \max _{\bar{\alpha} \in I \backslash\{\bar{\beta}\}}\left(8^{p_{\bar{\alpha}}+1} \sum_{i=1}^{\infty}\left(1-\left|b_{i, \bar{\alpha}}\right|\right)^{p_{\bar{\alpha}}+1}\right)\right)$

with $p_{\bar{\beta}}$ the smallest positive integer for which $\sum_{i=1}^{\infty}\left(1-\left|c_{i, \bar{\beta}}\right|\right)^{p_{\bar{\beta}}+1}$ is finite $\left(\left\{c_{i, \bar{\beta}}\right\}\right.$ is the sequence of zeros of $\left.1 / a_{\bar{\beta}}\right)$ and $p_{\bar{\alpha}}$ the smallest positive integer for which $\sum_{i=1}^{\infty}\left(1-\left|b_{i, \bar{\alpha}}\right|\right)^{p_{\bar{\alpha}+1}}$ is finite $\left(\left\{b_{i, \bar{\alpha}}\right\}\right.$ is the sequence of zeros of $\left.a_{\bar{\alpha}}\right)$,

(iii) the zeros of $a_{\bar{\beta}}$ and the poles of $a_{\bar{\alpha}}$ are all located in the sector $\Omega$, where $\Omega=$ $\{z \mid a+\varepsilon<\arg z<b-\varepsilon$, where $0<b-a<2 \pi$ and $0<\varepsilon<(2 \pi-(b-a)) / 3\}$, 
(iv) there exists a number $\gamma$ with $0 \leq \gamma<q-K\left(\hat{\mu}+v_{\beta}+6\right)$ such that for all $z \in D$ with $1 / 2 \leq|z|<1$,

$$
\left|\frac{h_{\bar{\alpha}}(z)}{h_{\bar{\beta}}(z)}\right|=O\left(\frac{1}{(1-|z|)^{\gamma}}\right) \quad(r \longrightarrow 1)
$$

where $a_{\bar{\beta}}(z)=z^{l(\bar{\beta})} P_{\bar{\beta}}(z) h_{\bar{\beta}}(z)$ with $P_{\bar{\beta}}(z)$ the Tsuji canonical product of the zeros of $a_{\bar{\beta}}$ and $a_{\bar{\alpha}}(z)=h_{\bar{\alpha}}(z) / z^{l(\bar{\alpha})} P_{\bar{\alpha}}(z)$ with $P_{\bar{\alpha}}(z)$ the Tsuji canonical product for the zeros of $1 / a_{\bar{\alpha}}$,

(v) there is a sector $\Lambda=\{z / c<\arg z<d, 0<d-c<2 \pi\}$ with $\Lambda \cap \tilde{\Omega}=\varnothing$ for which

$$
\iint_{\arg (z) \notin \Lambda}^{|z| \leq r}\left|f^{\#}(z)\right|^{2} d x d y=O\left(\frac{1}{1-r}\right) \quad(r \longrightarrow 1)
$$

where $\tilde{\Omega}=\{z \mid a<\arg z<b$, a and $b$ as in (iii) $\}$.

Then

$$
\limsup _{r \rightarrow 1} \frac{\log T_{0}(r, f)}{-\log (1-r)}<\infty
$$

The arguments in our proofs proceed by contradiction and involve the use of normal families.

We will present a proof for Theorem 2.1 in Section 3 and a proof of Theorem 2.3 in Section 4 . The proof for Theorem 2.2 proceeds along similar lines and will be omitted.

3. Proof of Theorem 2.1. We will use a lemma attributed to Zalcman [6, 7].

LEMMA 3.1. A family $\mathfrak{I}$ of meromorphic functions on the unit disk is not normal if and only if there exist a number $0<r<1$, points $z_{k},\left|z_{k}\right|<r$, functions $f_{k} \in \mathfrak{I}$, and positive real numbers $\rho_{k} \rightarrow 0$ such that $f_{k}\left(z_{k}+\rho_{k} \zeta\right)$ converges spherically uniformly on compact subsets of $\mathbb{C}$ to a nonconstant meromorphic function $g(\zeta)$. The function $g$ may be taken to satisfy the normalization $\mathfrak{g}^{\#}(z) \leq \mathfrak{g}^{\#}(0)=1, z \in \mathbb{C}$.

We proceed with the proof of Theorem 2.1 by assuming there exists a solution $f$ for our equation with

$$
\limsup _{r \rightarrow 1} \frac{\log T_{0}(r, f)}{-\log (1-r)}=\infty
$$

This implies that for any $A>0$, there exists a sequence $r_{k} \rightarrow 1$ such that $\log T_{0}\left(r_{k}, f\right) /$ $-\log \left(1-r_{k}\right)>A$ for all $k \geq N_{0}$.

We claim that there exists a sequence $w_{k},\left|w_{k}\right|=r_{k} \rightarrow 1$, such that

$$
\left(1-\left|w_{k}\right|\right)^{A / 2+1} f^{\#}\left(w_{k}\right) \rightarrow \infty
$$

for all $k \geq N_{0}$. 
Otherwise,

$$
\begin{aligned}
S(t) & =\frac{1}{\pi} \iint_{|z|<t}\left|f^{\#}(z)\right|^{2} d x d y \\
& =\frac{1}{\pi} \iint_{|z|<t} O\left(\frac{1}{(1-|z|)^{A+2}}\right) d x d y \\
& =O\left(\left(\frac{1}{1-t}\right)^{A+1}\right) .
\end{aligned}
$$

So,

$$
T_{0}\left(r_{k}, f\right)=\int_{0}^{r_{k}} \frac{S(t)}{t} d t=O\left(\left(\frac{1}{1-r_{k}}\right)^{A}\right)
$$

a contradiction.

Therefore, for $k \geq N_{0}$,

$$
f^{\#}\left(w_{k}\right)>\frac{1}{\left(1-r_{k}\right)^{A / 2+1}}
$$

Now, consider the family $\mathfrak{I}=\left\{f_{k}\right\}$ in the unit disk, where

$$
f_{k}(z)=f\left(\frac{z+w_{k}}{1+\bar{w}_{k} z}\right)
$$

Note that $\phi(z)=\left(z+w_{k}\right) /\left(1+\bar{w}_{k} z\right)$ maps the unit disk $D$ conformally onto itself. Taking the derivative with respect to $z$ gives

$$
f_{k}^{\prime}(z)=\frac{1-\left|w_{k}\right|^{2}}{\left(1+\bar{w}_{k} z\right)^{2}} f^{\prime}\left(\frac{z+w_{k}}{1+\bar{w}_{k} z}\right),
$$

and so

$$
f_{k}^{\prime}(0)=\left(1-\left|w_{k}\right|^{2}\right) f^{\prime}\left(w_{k}\right) .
$$

Hence,

$$
\begin{aligned}
f_{k}^{\#}(0) & =\frac{\left|f_{k}^{\prime}(0)\right|}{1+\left|f_{k}(0)\right|^{2}} \\
& =\left(1-\left|w_{k}\right|^{2}\right) f^{\#}\left(w_{k}\right) .
\end{aligned}
$$

So,

$$
f^{\#}(0)>\frac{1}{\left(1-r_{k}\right)^{A / 2}} .
$$

Thus, as $k \rightarrow \infty$,

$$
f_{k}^{\#}(0) \longrightarrow+\infty
$$


Hence, $\mathfrak{I}$ is not a normal family by Marty's criterion. By Lemma 3.1, there exist a real number $0<r<1$, a sequence of complex numbers $\left\{z_{k}\right\}$ in $D,\left|z_{k}\right|<r$, such that $z_{k} \rightarrow 0$, a sequence $\left\{\rho_{k}\right\}$ of positive real numbers such that $\rho_{k} \rightarrow 0^{+}$, and a nonconstant meromorphic function $g$ in $\mathbb{C}$ such that

$$
f_{k}\left(z_{k}+\rho_{k} \zeta\right)=f\left(\frac{\left(z_{k}+\rho_{k} \zeta\right)+w_{k}}{1+\bar{w}_{k}\left(z_{k}+\rho_{k} \zeta\right)}\right)=g_{k}(\zeta) \longrightarrow g(\zeta)
$$

as $k \rightarrow \infty$, spherically uniformly on compact subsets of $\mathbb{C} . g_{k}$ is defined on the compact sets $|\zeta| \leq\left(r-\left|z_{k}\right|\right) / \rho_{k}$. In the construction of the proof of Lemma 3.1,

$$
\begin{gathered}
\rho_{k}=\frac{1}{f_{k}^{\#}\left(z_{k}\right)}, \\
g_{k}^{\#}(0) \geq g_{k}^{\#}\left(-\frac{z_{k}}{\rho_{k}}\right) .
\end{gathered}
$$

Therefore, we have

$$
f_{k}^{\#}\left(z_{k}\right)=g_{k}^{\#}(0) \geq g_{k}^{\#}\left(-\frac{z_{k}}{\rho_{k}}\right)=f^{\#}\left(w_{k}\right)=f_{k}^{\#}(0)>\frac{1}{\left(1-r_{k}\right)^{A / 2}},
$$

so

$$
\rho_{k}=\frac{1}{f_{k}^{\#}\left(z_{k}\right)}<\left(1-r_{k}\right)^{A / 2}
$$

and $\rho_{k} \rightarrow 0$ as $k \rightarrow \infty$.

Now, since $a_{\bar{\beta}}(z)$ is not identically zero, we can divide both sides of (1.1) through by $a_{\bar{\beta}}(z)$, and write it in the form

$$
f^{\prime}(z)^{\beta_{1}}=-\sum_{\bar{\alpha} \in I \backslash\{\bar{\beta}\}} \frac{a_{\bar{\alpha}}(z)}{a_{\bar{\beta}}(z)} f(z)^{\alpha_{0}-\beta_{0}} f^{\prime}(z)^{\alpha_{1}} f^{(2)}(z)^{\alpha_{2}-\beta_{2}} \cdots f^{(m)}(z)^{\alpha_{m}-\beta_{m}} .
$$

We proceed to substitute

$$
\tau_{k}=\phi\left(z_{k}+\rho_{k} \zeta\right)=\frac{\left(z_{k}+\rho_{k} \zeta\right)+w_{k}}{1+\bar{w}_{k}\left(z_{k}+\rho_{k} \zeta\right)}
$$

for $z$ into the differential equation. We have

$$
\begin{aligned}
g_{k}^{\prime}(\zeta) & =\frac{d}{d \zeta} f\left(\frac{\left(z_{k}+\rho_{k} \zeta\right)+w_{k}}{1+\bar{w}_{k}\left(z_{k}+\rho_{k} \zeta\right)}\right) \\
& =\frac{\rho_{k}\left(1-\left|w_{k}\right|^{2}\right)}{\left(1+\bar{w}_{k}\left(z_{k}+\rho_{k} \zeta\right)\right)^{2}} f^{\prime}\left(\frac{\left(z_{k}+\rho_{k} \zeta\right)+w_{k}}{1+\bar{w}_{k}\left(z_{k}+\rho_{k} \zeta\right)}\right)
\end{aligned}
$$

Differentiating with respect to $\zeta$, we obtain

$$
\begin{aligned}
& \left(1-\left|w_{k}\right|^{2}\right)^{2} \rho_{k}^{2} f^{\prime \prime}\left(\tau_{k}\right) \\
& \quad=\left(1+\bar{w}_{k}\left(z_{k}+\rho_{k} \zeta\right)\right)^{4} g_{k}^{\prime \prime}(\zeta)+2 \bar{w}_{k} \rho_{k}\left(1+\bar{w}_{k}\left(z_{k}+\rho_{k} \zeta\right)\right)^{3} g_{k}^{\prime}(\zeta),
\end{aligned}
$$


and by induction we can show that for $j=1,2,3, \ldots$,

$$
f^{(j)}\left(\tau_{k}\right)=\left[\left(1-\left|w_{k}\right|^{2}\right) \rho_{k}\right]^{-j} H_{j}\left[g_{k}\right](\zeta),
$$

where $H_{j}\left[g_{k}\right]$ is a polynomial function of the derivatives $g_{k}^{\prime}, \ldots, g_{k}^{(j)}$, which converges to $H_{j}[g]$ as $k \rightarrow \infty$. Using (3.20) in (3.16), we get

$$
\begin{aligned}
& {\left[\frac{\left(1+\bar{w}_{k}\left(z_{k}+\rho_{k} \zeta\right)\right)^{2} g_{k}^{\prime}(\zeta)}{\left(1-\left|w_{k}\right|^{2}\right) \rho_{k}}\right]^{\beta_{1}}} \\
& \quad=-\sum_{\bar{\alpha} \in I \backslash\{\bar{\beta}\}} \frac{a_{\bar{\alpha}}\left(\tau_{k}\right)}{a_{\bar{\beta}}\left(\tau_{k}\right)}\left[\left(1-\left|w_{k}\right|^{2}\right) \rho_{k}\right]^{-\left[\alpha_{1}+2\left(\alpha_{2}-\beta_{2}\right)+\cdots+m\left(\alpha_{m}-\beta_{m}\right)\right]} M_{\bar{\alpha}}\left[g_{k}\right](\zeta),
\end{aligned}
$$

where

$$
M_{\bar{\alpha}}\left[g_{k}\right](z)=H_{0}^{\alpha_{0}}\left[g_{k}\right](z) H_{1}^{\alpha_{1}}\left[g_{k}\right](z) H_{2}^{\alpha_{2}}\left[g_{k}\right](z) \cdots H_{m}^{\alpha_{m}}\left[g_{k}\right](z)
$$

Multiplying both sides of the equality by $\left[\left(1-\left|w_{k}\right|^{2}\right) \rho_{k}\right]^{\beta_{1}}$, where $\beta_{1}$ is assumed to be a positive integer, we get

$$
\left[\left(1+\bar{w}_{k}\left(z_{k}+\rho_{k} \zeta\right)\right)^{2} g_{k}^{\prime}(\zeta)\right]^{\beta_{1}}=-\sum_{\bar{\alpha} \in I \backslash\{\bar{\beta}\}} \frac{a_{\bar{\alpha}}\left(\tau_{k}\right)}{a_{\bar{\beta}}\left(\tau_{k}\right)}\left[\left(1-\left|w_{k}\right|^{2}\right) \rho_{k}\right]^{q} M_{\bar{\alpha}}\left[g_{k}\right](\zeta)
$$

The modulus of the right-hand side of (3.23) is less than

$$
K \sum_{\bar{\alpha} \in I \backslash\{\bar{\beta}\}} \frac{1}{\left(1-\left|\tau_{k}\right|\right)^{q}}\left[\left(1-\left|w_{k}\right|^{2}\right) \rho_{k}\right]^{q}\left|M_{\bar{\alpha}}\left[g_{k}\right](\zeta)\right|
$$

Now, we use the inequality

$$
\begin{aligned}
2\left(1-\left|\tau_{k}\right|\right) & \geq 1-\left|\tau_{k}\right|^{2} \\
& =1-\frac{\left|\left(z_{k}+\rho_{k} \zeta\right)-w_{k}\right|^{2}}{\left|1+\bar{w}_{k}\left(z_{k}+\rho_{k} \zeta\right)\right|^{2}} \\
& =\frac{\left|1+\bar{w}_{k}\left(z_{k}+\rho_{k} \zeta\right)\right|^{2}-\left|\left(z_{k}+\rho_{k} \zeta\right)-w_{k}\right|^{2}}{\left|1+\bar{w}_{k}\left(z_{k}+\rho_{k} \zeta\right)\right|^{2}} \\
& \geq \frac{1+\left|\bar{w}_{k}\left(z_{k}+\rho_{k} \zeta\right)\right|^{2}-\left|z_{k}+\rho_{k} \zeta\right|^{2}-\left|\bar{w}_{k}\right|^{2}}{4} \\
& =\frac{\left(1-\left|z_{k}+\rho_{k} \zeta\right|^{2}\right)\left(1-\left|\bar{w}_{k}\right|^{2}\right)}{4} .
\end{aligned}
$$


So, since $\left|z_{k}+\rho_{k} \zeta\right| \rightarrow 0$, as $k \rightarrow \infty$, there exists an integer $N_{1}$ such that $\left|z_{k}+\rho_{k} \zeta\right| \leq 1 / 3$ for all $k \geq N_{1}$. Therefore, for such $k$,

$$
\begin{gathered}
\frac{1-\left|w_{k}\right|^{2}}{1-\left|\tau_{k}\right|} \leq 9, \\
\left(\frac{1-\left|w_{k}\right|^{2}}{1-\left|\tau_{k}\right|}\right)^{q} \leq 9^{q} .
\end{gathered}
$$

Thus, the right-hand side of (3.23) can be bounded in modulus by

$$
9^{q} K \sum_{\bar{\alpha} \in I \backslash\{\bar{\beta}\}} \rho_{k}^{q}\left|M_{\bar{\alpha}}\left[g_{k}\right](\zeta)\right|
$$

which goes to zero as $k \rightarrow \infty$.

But as $k \rightarrow \infty$, the left-hand side of (3.23) goes to $g^{\prime}(\zeta)^{\beta_{1}}$. Hence, we obtain $\left(g^{\prime}(\zeta)\right)^{\beta_{1}} \equiv$ 0 in contradiction to $g$ being a nonconstant function.

4. Proof of Theorem 2.3. We will use the following lemma which is a modification of [4, Theorem V.25, page 224].

LEMMA 4.1. Let $f$ be a meromorphic function of finite order in $D$, and let $\left\{a_{n}\right\}$ be its zero points for which $a_{n} \neq 0$. Let $P$ be the Tsuji canonical product formed with $\left\{a_{n}\right\}$, and let $\mu$ be the convergence exponent of $\left\{\left|a_{n}\right|\right\}$. For $n=1,2,3, \ldots$, denote by $C_{n}$ the circle $\left|z-a_{n}\right|=\left(1-\left|a_{n}\right|^{2}\right)^{\mu+4}$. If $z$ lies outside of $C_{n}$ for $n=1,2,3, \ldots$ and $1 / 2 \leq|z|<1$, then

$$
\log ^{+} \frac{1}{|P(z)|} \leq \log \left(\frac{2^{\mu+4}}{r_{0}} \cdot \frac{1}{(1-|z|)^{p+1}}\right) 8^{p+1} \sum_{n}\left(1-\left|a_{n}\right|\right)^{p+1},
$$

where $r_{0}=\min \left|a_{n}\right|$ and $p$ is a positive integer such that $\sum_{n}\left(1-\left|a_{n}\right|\right)^{p}=\infty$ and $\sum_{n}(1-$ $\left.\left|a_{n}\right|\right)^{p+1}<\infty$.

To prove Theorem 2.3, we argue by contradiction assuming first that there exists a solution $f$ to the differential equation with

$$
\limsup _{r \rightarrow 1} \frac{\log T_{0}(r, f)}{-\log (1-r)}=\infty
$$

Then we claim that for each $A \geq 1$ there exists a sequence $w_{k}$ with $\arg w_{k}$ in $S$, and $\left|w_{k}\right|=r_{k} \rightarrow 1$, such that

$$
\left(1-\left|w_{k}\right|\right)^{A / 2+1} f^{\#}\left(w_{k}\right) \longrightarrow \infty
$$

for $k \rightarrow \infty$. Otherwise, for $0<t<1$,

$$
\lambda(t)=\frac{1}{\pi} \iint_{\substack{|z|<t \\ \arg z \in \Omega}}\left|f^{\#}(z)\right|^{2} d x d y \leq \frac{1}{\pi} \iint_{\substack{|z|<t \\ \arg z \in \Omega}} \frac{K}{(1-|z|)^{A+2}} d x d y
$$


for some constant $K$. Hence

$$
\lambda(t)=O\left(\left(\frac{1}{1-t}\right)^{A+1}\right) \quad(t \rightarrow 1) .
$$

Combining this estimate with assumption (v), we have

$$
T_{0}(r, f)=\int_{0}^{r} \frac{S(t)}{t}=O\left(\left(\frac{1}{1-r}\right)^{A}\right) \quad(r \longrightarrow 1)
$$

which contradicts (4.2).

Therefore the sequence $\left\{w_{k}\right\}$ exists and there is an integer $N_{0}$ such that for $k \geq N_{0}$,

$$
f^{\#}\left(w_{k}\right)>\frac{1}{\left(1-r_{k}\right)^{A / 2+1}} .
$$

As in the proof of Theorem 2.1, we observe that Marty's criterion shows that the family $\mathfrak{I}=\left\{f_{k}\right\}$ defined in the unit disk by

$$
f_{k}(z)=f\left(\frac{z+w_{k}}{1+\bar{w}_{k} z}\right)
$$

is not a normal family.

Also, by Lemma 3.1, there are a real number $r$ with $0<r<1$, a sequence of complex numbers $\left\{z_{k}\right\}$ in $D$ with $\left|z_{k}\right|<r$ such that $z_{k} \rightarrow 0$ as $k \rightarrow \infty$, a sequence $\left\{\rho_{k}\right\}$ of positive real numbers such that $\rho_{k} \rightarrow 0^{+}$as $k \rightarrow \infty$, and a nonconstant function $g$ in $\mathbb{C}$ such that

$$
f_{k}\left(z_{k}+\rho_{k} \zeta\right)=f\left(\frac{\left(z_{k}+\rho_{k} \zeta\right)+w_{k}}{1+\bar{w}_{k}\left(z_{k}+\rho_{k} \zeta\right)}\right)=g_{k}(\zeta) \rightarrow g(\zeta)
$$

as $k \rightarrow \infty$, spherically uniformly on compact subsets of $\mathbb{C} . g_{k}$ is defined on compact sets $\left\{\zeta /|\zeta| \leq\left(r-\left|z_{k}\right|\right) / \rho_{k}\right\}$. Further, the proof of Lemma 3.1 gives $\rho_{k}=1 / f^{\#}\left(z_{k}\right)$.

Since $a_{\bar{\beta}}$ is not identically zero, we can divide (1.1) through by $a_{\bar{\beta}}(z)$ and write it in the form (3.16). Proceeding as in the proof of Theorem 2.1 with the substitution of $\tau_{k}$ for $z$ in the differential equation, the differentiation with respect to $\zeta$, and the induction process for the derivatives of $f$ at $\tau_{k}$, we again obtain (3.23). Replacing $a_{\bar{\alpha}}$ and $a_{\bar{\beta}}$ by their representatives in assumption (iv), we get

$$
\begin{aligned}
& {\left[\left(1+\bar{w}_{k}\left(z_{k}+\rho_{k} \zeta\right)\right)^{2} g_{k}^{\prime}(\zeta)\right]^{\beta_{1}}} \\
& \quad=\sum_{\bar{\alpha} \in I \backslash\{\bar{\beta}\}} \frac{-h_{\bar{\alpha}}\left(\tau_{k}\right)}{h_{\bar{\beta}}\left(\tau_{k}\right)} \frac{\left[\left(1-\left|w_{k}\right|^{2}\right) \rho_{k}\right]^{q}}{\tau_{k}^{l(\bar{\alpha})+l(\bar{\beta})} P_{\bar{\alpha}}\left(\tau_{k}\right) P_{\bar{\beta}}\left(\tau_{k}\right)} M_{\bar{\alpha}}\left[g_{k}\right](\zeta) .
\end{aligned}
$$

Assumptions (iii) and (iv) assure that Lemma 4.1 may be used to estimate $1 / P_{\bar{\alpha}}\left(\tau_{k}\right) P_{\bar{\beta}}\left(\tau_{k}\right)$ for which we obtain

$$
\frac{1}{\left|P_{\bar{\alpha}}\left(\tau_{k}\right) P_{\bar{\beta}}\left(\tau_{k}\right)\right|} \leq \hat{K}\left(\frac{1}{1-\left|\tau_{k}\right|}\right)^{K\left(\hat{\mu}+v_{\beta}+6\right)}
$$


for each pair of $\bar{\alpha}$ and $\bar{\beta}$, where $\hat{K}$ is a constant independent of $k$. Thus, by assumption (iv), the modulus of the right-hand side of (4.10) is bounded by

$$
K_{0} \sum_{\bar{\alpha} \in I \backslash\{\bar{\beta}\}} \frac{1}{\left(1-\left|\tau_{k}\right|\right)^{\gamma}} \frac{\left[\left(1-\left|w_{k}\right|^{2}\right) \rho_{k}\right]^{q}}{\left|\tau_{k}\right|^{l(\bar{\alpha})+l(\bar{\beta})}} \frac{\left|M_{\bar{\alpha}}\left[g_{k}\right](\zeta)\right|}{\left(1-\left|\tau_{k}\right|\right)^{K\left(\hat{\mu}+v_{\beta}+6\right)}},
$$

where $K_{0}$ is a constant.

As in the proof of Theorem 2.1, we have

$$
1-\left|w_{k}\right|^{2} \leq 9\left(1-\left|\tau_{k}\right|\right)
$$

so our estimate becomes

$$
K_{0} \sum_{\bar{\alpha} \in I \backslash\{\bar{\beta}\}} \rho_{k}^{q}\left(1-\left|\tau_{k}\right|\right)^{q-\gamma-K\left(\hat{\mu}+v_{\beta}+6\right)} \frac{\left|M_{\bar{\alpha}}\left[g_{k}\right](\zeta)\right|}{\left|\tau_{k}\right|^{l(\bar{\alpha})+l(\bar{\beta})}} .
$$

Hence, as $k$ goes to infinity, the modulus of the right-hand side of (4.10) goes to zero, and since the left-hand side goes to $\left|g^{\prime}(\zeta)\right|^{\beta_{1}}$, we have a contradiction.

REMARK 4.2. A more refined theorem of the nature of Theorem 2.3 appears in the dissertation of Benbourenane [2].

\section{REFERENCES}

[1] S. B. Bank, A general theorem concerning the growth of solutions of first-order algebraic differential equations, Compositio Math. 25 (1972), 61-70.

[2] D. Benbourenane, Value distribution for solutions of complex differential equations in the unit disk, Dissertation, Northern Illinois University, Illinois, 2001.

[3] J. Heittokangas, On complex differential equations in the unit disc, Ann. Acad. Sci. Fenn. Math. Diss. (2000), no. 122, 1-54.

[4] M. Tsuji, Potential Theory in Modern Function Theory, Maruzen, Tokyo, 1959.

[5] H. Wulan, On some classes of meromorphic functions, Ann. Acad. Sci. Fenn. Math. Diss. (1998), no. 116, 1-57.

[6] L. Zalcman, A heuristic principle in complex function theory, Amer. Math. Monthly 82 (1975), no. 8, 813-817.

[7] _ Normal families: new perspectives, Bull. Amer. Math. Soc. (N.S.) 35 (1998), no. 3, 215-230.

D. Benbourenane: Department of Mathematics and Computer Science, United Arab Emirates University, P.O. Box 17551, Al-Ain, United Arab Emirates

E-mail address: d. benbourenane@uaeu .ac.ae

L. R. Sons: Department of Mathematical Sciences, Northern Illinois University, DeKalb, IL 601152888, USA

E-mail address: sons@math.niu.edu 


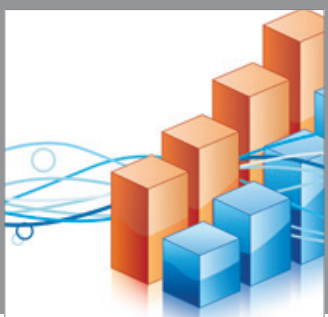

Advances in

Operations Research

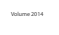

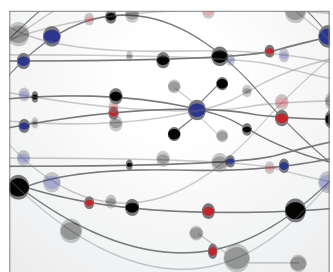

\section{The Scientific} World Journal
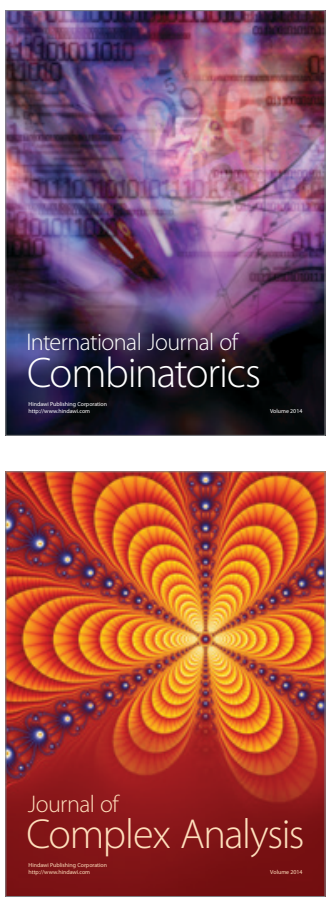

International Journal of

Mathematics and

Mathematical

Sciences
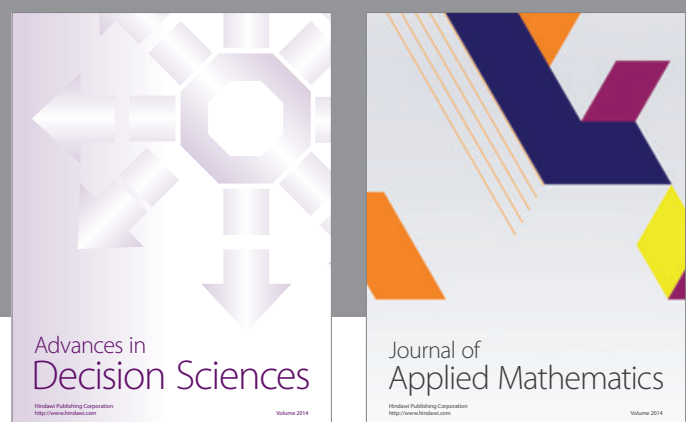

Journal of

Applied Mathematics
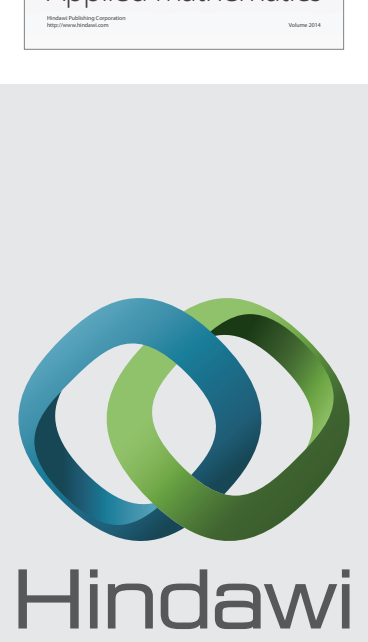

Submit your manuscripts at http://www.hindawi.com
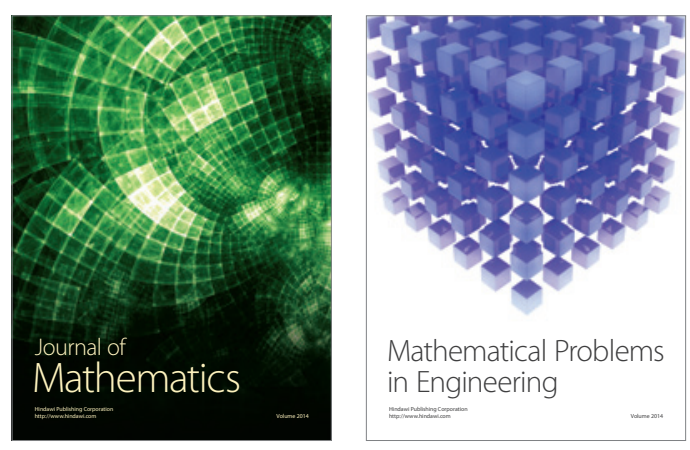

Mathematical Problems in Engineering
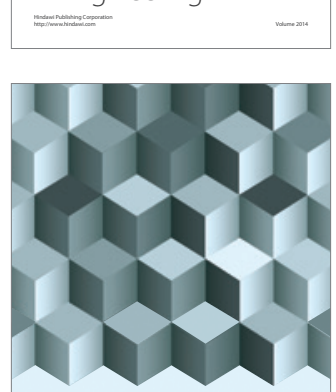

Journal of

Function Spaces
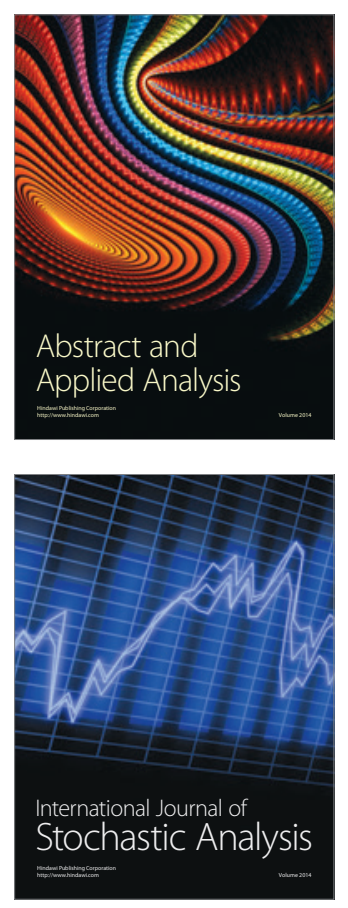

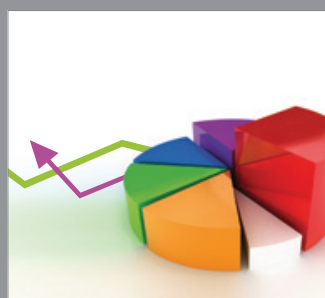

ournal of

Probability and Statistics

Promensencen
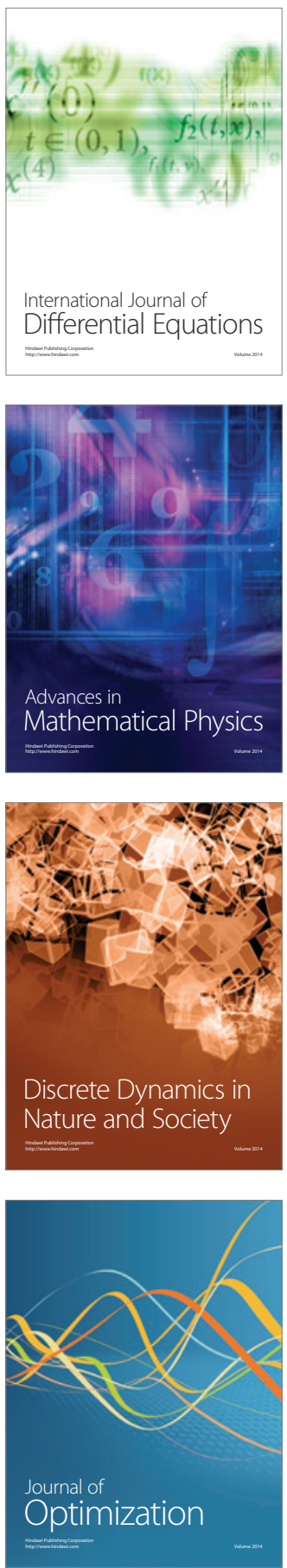